\title{
Learning Action Selection Network of Intelligent Agent
}

\author{
Eun-Kyung Yun and Sung-Bae Cho \\ Department of Computer Science, Yonsei University \\ 134 Shinchon-dong, Sudaemoon-ku, Seoul 120-749, Korea \\ ekfree@candy.yonsei.ac.kr, sbcho@cs.yonsei.ac.kr \\ http://sclab.yonsei.ac.kr
}

\begin{abstract}
Behavior-based artificial intelligent system is to derive the complicated behaviors by selecting appropriate one from a set of basic behaviors. Many robot systems have used behavior-based systems since the 1980's. In this paper, we propose new method to create the sequences of behaviors appropriate to the changing environments by adding the function of learning with Learning Classifier System to P. Maes' action selection network. Links of the network need to be reorganize as the problem changes, because each link is designed initially according to the given problem and is fixed. Learning Classifier System is suitable for learning of rule-based system in changing environments. The simulation results with Khepera robot simulator show the usefulness of learning in the action selection network by generating appropriate behaviors.
\end{abstract}

\section{Introduction}

In behavior-based artificial intelligent system, the architecture is not to compose of sequential modules, such as perception, modeling, planning, and task execution, but to derive more complicated behaviors by selecting appropriate one from a set of basic behaviors [1] [2]. Tinbergen has proposed Hierarchical Decision Structure for action selection [3]. There are some related publications such as Brooks' Subsumption Architecture [4], Tyrrell's hierarchical structure [5], and Mataric's group studying learning via human behavior imitation [6].

Learning in robots is known to be a difficult problem, due to the uncertainty in sensing and action, partial observability of the robot's environment, and non-stationarity of the world. Learning in behavior-based system focuses on the efficiency improved in short time instead of long optimization [7]. Effective action selection is a matter of primary concern in behavior-based system. It can be easily supported by using the well-known reinforcement learning whose policy selects the action that maximizes rewards. Some examples of reinforcement learning in the context of behavior-based system demonstrated hexapod walking [1] and box-pushing [8]. Besides in multi-robot systems, M. Dorigo and M. Colombetti [9] applied the idea of Shaping to the original reinforcement learning.

In this paper, we propose the new dynamic action selection network adding Learning Classifier System (LCS) in order to adapt to the changing environment 


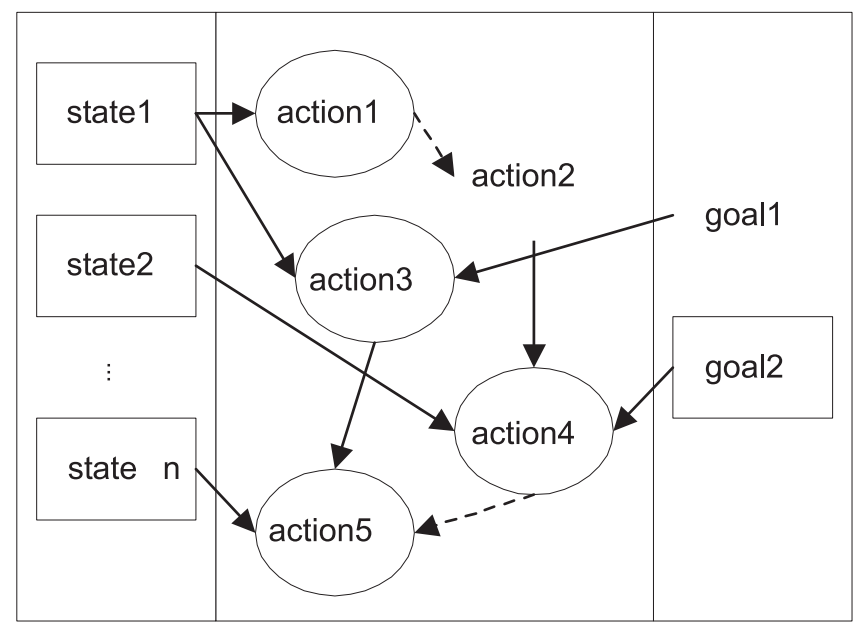

Fig. 1. The structure of the action selection network

like the real world. Maes' network has a shortcoming of selecting inappropriate actions when the task changes because it is difficult to modify the structure. In order to solve this problem, we improve the network appropriate to the changing environment by applying the function of learning.

\section{Action Selection Network}

Maes' action selection network is a distributed, non-hierarchical network [10]. It consists of action nodes, motivation or goal nodes, and environmental state nodes (see Fig. 1). These nodes have the different types of links that encode various relationships and stimulate each other. The compositions of a node are preconditions, add list, delete list, activation level, and the code that is run if the node is executed. Fig. 2 shows the elements of a node in action selection network.

If the proposition $\mathrm{X}$ about environment is true and $\mathrm{X}$ is in the precondition list of node $\mathrm{A}$, there is an active link from the state node $\mathrm{X}$ to action node $\mathrm{A}$. If goal $\mathrm{Y}$ has an activation greater than zero and $\mathrm{Y}$ is in the add list of node $\mathrm{A}$, there is an active link from goal node $\mathrm{Y}$ to action node $\mathrm{A}$. Internal links include predecessor links, successor links, and conflicter links. Fig. 3 shows the conditions of each internal link.

The following is the procedure to select an action node to be executed at each step.

1. Calculate the excitation coming in from the environment and the motivations. 
2. Spread excitation along the predecessor, successor, and conflicter links, and normalize the node activations so that the average activation becomes equal to the constant $\pi$.

3. Check any executable nodes, choose the one with the highest activation, execute it, and finish. A node is executable if all the preconditions are true and if its activation is greater than the global threshold. If no node is executable, reduce the global threshold and repeat the cycle.

Every link is established manually by a designer according to the initial task given. Therefore it can solve the initial task completely but it needed to be reconstructed according to that task when the task changes. In order to solve this problem, the robot itself has to learn to construct the network and reorganize all links. To change the robot tasks means to change the status of robot. The robot has to consider the changed conditions and select the proper actions.

\section{Learning Action Selection Network with LCS}

In machine learning, neural networks and genetic algorithm are widely used. However, in this paper, learning classifier system is used for learning of the action selection network. It is the learning algorithm for rule-based system and is appropriate to this case. The state nodes and goal nodes, which are elements of action selection network, can be considered as a kind of conditions. Therefore these are easy to convert to the rule-based system. This is the reason that we select the learning classifier system for learning.

\subsection{LCS (Learning Classifier System)}

LCS, a kind of machine learning technique, was introduced by Holland and Reitman in 1978 [12]. LCS has two different learning procedures. One is to learn via mixing given rules (Credit Assignment System) and the other is to learn via creating useful rules as possible (Rule Discovery System). It is very appropriate

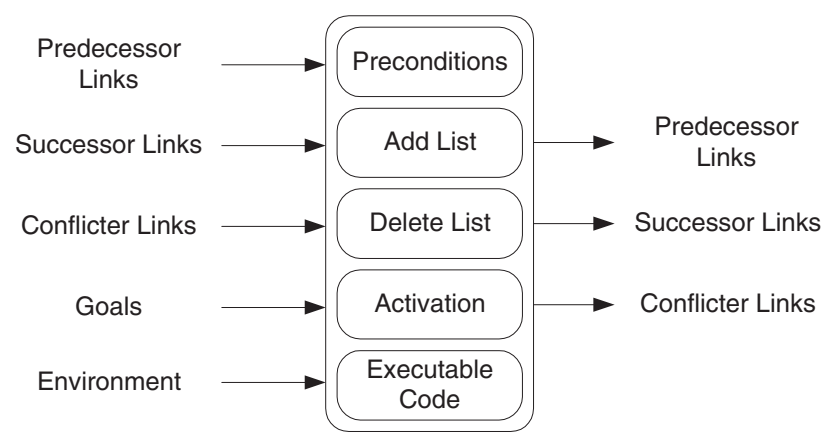

Fig. 2. The elements of a node in action selection network 


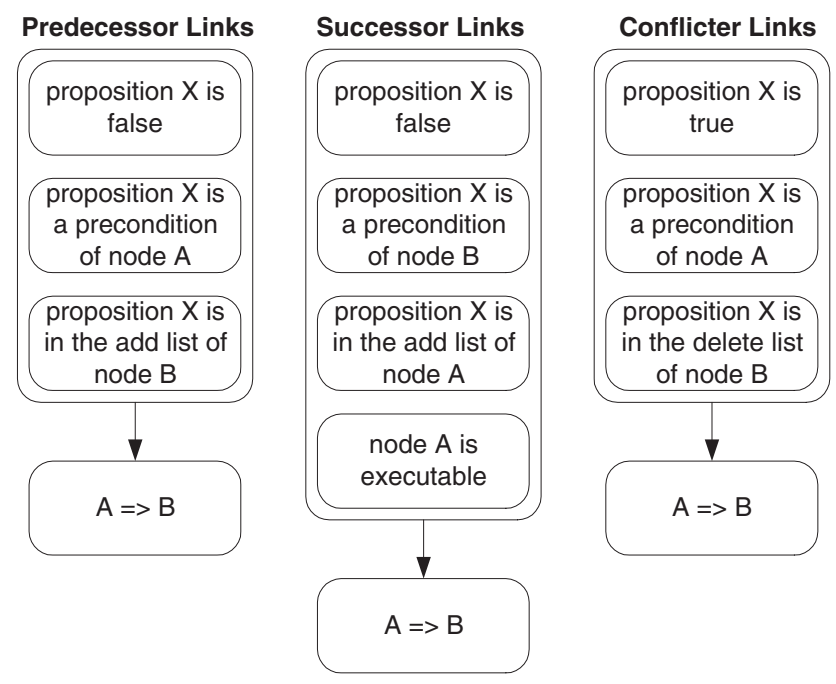

Fig. 3. The internal links

to be adapted to a changing environment. Classifier system consists of several rules, so-called classifiers. One classifier has one or more condition parts and one action part. The condition of a classifier consists of ternary elements $\{0,1, \#\}$ and the action part consists of $\{0,1\}$. The character '\#' means "don't care" and can take either ' 0 ' or ' 1 '.

In the competition of classifiers, the value of strength gives a measure of the rule's past performance. That is, the higher a classifier's strength the better it has performed. In addition, each classifier has the value of specificity, which is the number of non-\# symbols in the condition part. LCS consists of three modules as shown in Fig. 4.

- Classifier System: The system compares input messages with the condition part of all classifiers in the classifier list and performs matching. This acts by bit-to-bit. The matching classifiers enter competition for the right to post their output messages, and only the winner of the competition actually posts messages. The measure of each classifier is the value of bid as follows:

$$
\text { bid }=c \times \text { specificity } \times \text { strength }
$$

where c: a constant less than 1, specificity: specificity of the classifier condition, condition's length minus the number of '\#' symbols, and strength: the measure of confidence in a classifier. When feedback comes from its environment, the strength of the winner is recomputated.

- Credit Assignment System: When the rewards from the environment transmit, they cause the strength of the classifiers to change in order to reflect their relevance to the system performance. This system is to classify rules 


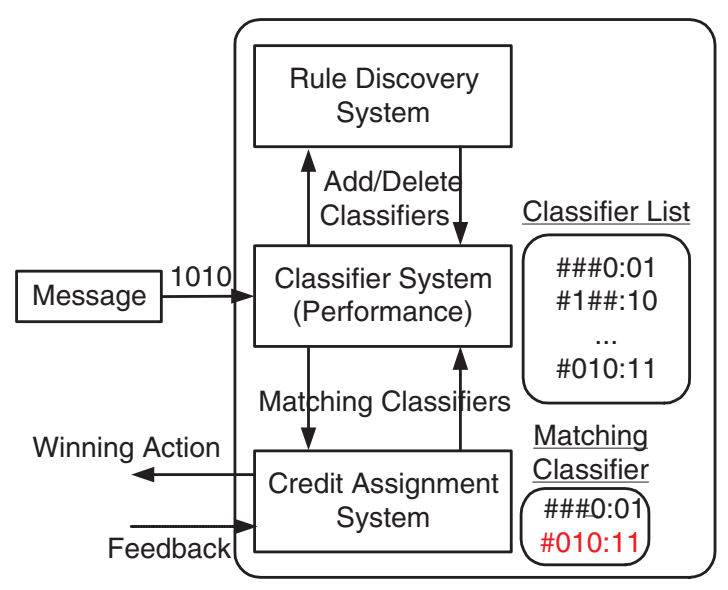

Fig. 4. The structure of LCS

in accordance with their usefulness. The classical algorithm used for this purpose is the Bucket Brigade algorithm [12].

- Rule Discovery System: The system uses the genetic algorithms to discover new rules. Genetic algorithms are stochastic algorithms that have been used both as optimization and as rule discovery mechanism. They work modifying a population of solutions (in LCS, a solution is a classifier). Solutions are properly coded and a fitness function is defined to relate solutions to performance. The value from this function is a measure of the solution quality. The fitness of a classifier is set by its usefulness calculated with a credit apportionment system instead of a fitness function.

In general, while classifier system and credit apportionment system are interested in the classifiers with better performances, rule discovery system is interested in the classifiers with worse performances. If the only classifiers with high scores survive, the system cannot discover new classifiers.

\subsection{Learning with LCS}

Our goal is to improve the robot's ability to solve problems using LCS, a kind of learning methods, in the action selection network. Firstly the rules of LCS, the basic elements, have to be defined for learning links in the network. The rules include the state nodes, the extent of the problems, and links between the nodes. They do not include the action nodes. When the rule is fixed, or the network structure is decided, it calculates the activation level and selects the action node with the highest activation value. Fig. 5 shows the structure of the rule defined. The extent of the problems and state nodes are determined through the robot's current states from environment. 


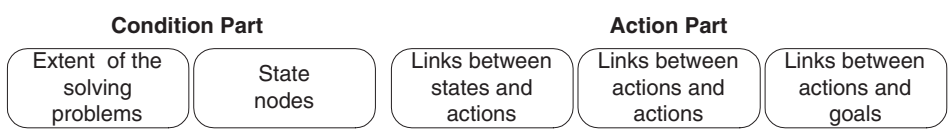

Fig. 5. The structure of the rule

While original action selection network includes three kinds of links between the nodes, we redefine the excitatory links for LCS. Excitatory links indicate predecessor links and successor links because they have similar meaning. In addition, the classifier lists are initialized in accordance with the purpose of tasks, not randomly. That is, the initial structure of network is converted into rules.

\section{Experimental Results}

\subsection{Experimental Environments}

The task of the robot is to reach the specific goal position in the shortest path. The network has only two actions of going straight and avoiding obstacles initially. Therefore the robot is going to complete the task using only two actions regardless of the goal position. However as shown in Fig. 6, other actions are better than the action of going straight if the robot considers where it exists. The initial network is converted into the rules, which form the classifier list, and the network is improved using LCS in order to select the proper action even though the position of robot changes.

Initial network is constructed as shown in Fig. 7 and Table 1 shows preconditions, add list elements, and delete list elements for each action node.

The learning of action selection network is tested on the Khepara robot simulator [13]. It has 8 distance sensors and light sensors. The distance sensors return the value ranging between 0 and 1023. 0 means that no object is perceived while 1023 means that an object is very close to the sensor. Intermediate values indicate approximate distance between the sensor and the object. Therefore we define the state node, 'obstacle around' is true if the distance value is more than 900 , and nothing around is true otherwise.
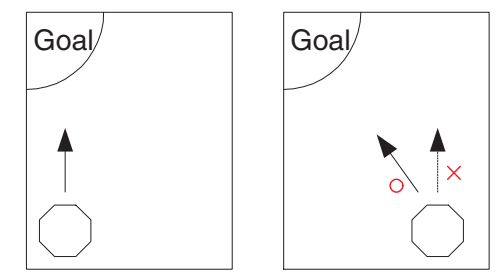

Fig. 6. The action selection for the position of robot 
Table 1. Preconditions, add list elements, and delete list elements for each action node

\begin{tabular}{|c|c|c|c|}
\hline Action node & Preconditions & Add list & Delete list \\
\hline Avoiding obstacles & $\begin{array}{c}\text { Obstacles } \\
\text { around }\end{array}$ & $\begin{array}{c}\text { Nothing } \\
\text { around }\end{array}$ & $\begin{array}{c}\text { Obstacles } \\
\text { around }\end{array}$ \\
\hline Going straight & $\begin{array}{c}\text { Nothing } \\
\text { around }\end{array}$ & $\begin{array}{c}\text { Obstacles } \\
\text { around }\end{array}$ & $\begin{array}{c}\text { Nothing } \\
\text { around }\end{array}$ \\
\hline $\begin{array}{c}\text { Turn left an angle of } \\
45 \text { degrees and go } \\
\text { straight }\end{array}$ & $\begin{array}{c}\text { Nothing } \\
\text { around }\end{array}$ & $\begin{array}{c}\text { Obstacles } \\
\text { around }\end{array}$ & $\begin{array}{c}\text { Nothing } \\
\text { around }\end{array}$ \\
\hline $\begin{array}{c}\text { Turn right an angle } \\
\text { of 45 degrees and go } \\
\text { straight }\end{array}$ & $\begin{array}{c}\text { Nothing } \\
\text { around }\end{array}$ & $\begin{array}{c}\text { Obstacles } \\
\text { around }\end{array}$ & $\begin{array}{c}\text { Nothing } \\
\text { around }\end{array}$ \\
\hline
\end{tabular}

Table 2 shows the composition of a classifier in LCS. Firstly the extent of solving problems is set by checking the location of the goal robot reaches. That is, 4 bits indicate up,down,right,left. For example, 1000 means the goal is upper and 1010 means the goal is left. In LCS, genetic operators act every 100 iterations.

\subsection{Results}

Before learning, the robot always begins to move by going straight as shown in Fig. 8. Therefore, it is impossible to reach the goal in the shortest path whenever the initial position of the robot changes. The goal of robot is the left upper corner and robot's initial position is set randomly in the experiments. The goal of robot is the left upper corner and robot's initial position is set randomly in the experiments. Many of the actions of going straight were selected before learning (see Fig. 8) but through the learning, other actions rather than the

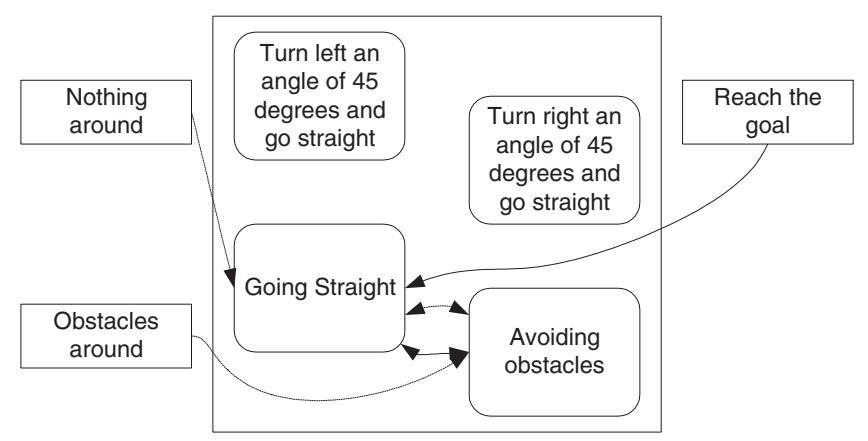

Fig. 7. The structure of initial network: Dotted lines mean inhibitory links and solid lines mean excitatory links in the internal links 
Table 2. The composition of a classifier

\begin{tabular}{|c|c|c|c|}
\hline & Bit & \multicolumn{2}{|c|}{ Descriptions } \\
\hline \multirow{6}{*}{\begin{tabular}{|c|} 
Condition \\
part \\
$(6$ bits $)$
\end{tabular}} & C1 & \multicolumn{2}{|c|}{ Position of goal = upper } \\
\hline & $\mathrm{C} 2$ & \multicolumn{2}{|c|}{ Position of goal = under } \\
\hline & $\mathrm{C} 3$ & \multicolumn{2}{|c|}{ Position of goal = left } \\
\hline & $\mathrm{C} 4$ & \multicolumn{2}{|c|}{ Position of goal = right } \\
\hline & C5 & \multicolumn{2}{|c|}{ Nothing around } \\
\hline & $\mathrm{C} 6$ & \multicolumn{2}{|c|}{ Obstacles around } \\
\hline \multirow{17}{*}{$\begin{array}{c}\text { Action part } \\
\text { (17 bits) }\end{array}$} & $\mathrm{A} 1$ & \multirow{2}{*}{\begin{tabular}{|c|} 
Links between \\
"Nothing around" \\
node and action \\
nodes, \\
11: Going straight, \\
10: Turn left, 01: \\
Turn right
\end{tabular}} & \multirow{3}{*}{$\begin{array}{c}\text { External links } \\
\text { (from sensors of the } \\
\text { environment, or } \\
\text { state nodes) }\end{array}$} \\
\hline & A2 & & \\
\hline & A3 & $\begin{array}{c}\text { Links between } \\
\text { "Obstacles around" } \\
\text { node and "Avoiding } \\
\text { obstacles" action } \\
\text { node }\end{array}$ & \\
\hline & A4 & \multirow{2}{*}{$\begin{array}{c}\text { Link from "Going } \\
\text { straight" to } \\
\text { "Avoiding obstacles" }\end{array}$} & \multirow{12}{*}{$\begin{array}{c}\text { Internal links } \\
(00: \text { no link, } \\
\text { 01: excitatory links, } \\
\text { 10: inhibitory links) }\end{array}$} \\
\hline & A5 & & \\
\hline & A6 & Link from "Avoiding & \\
\hline & A7 & $\begin{array}{c}\text { obstacles" to "Going } \\
\text { straight" }\end{array}$ & \\
\hline & A8 & \multirow{2}{*}{$\begin{array}{c}\text { Link from "Turn left" } \\
\text { to "Avoiding } \\
\text { obstacles" }\end{array}$} & \\
\hline & A9 & & \\
\hline & A10 & \multirow{2}{*}{$\begin{array}{c}\text { Link from "Avoiding } \\
\text { obstacles" to "Turn } \\
\text { left" }\end{array}$} & \\
\hline & A11 & & \\
\hline & $\mathrm{A} 12$ & \multirow{2}{*}{$\begin{array}{c}\text { Link from "Turn } \\
\text { right" to "Avoiding } \\
\text { obstacles" }\end{array}$} & \\
\hline & A13 & & \\
\hline & A14 & \multirow{2}{*}{$\begin{array}{c}\text { Link from "Avoiding } \\
\text { obstacles" to "Turn } \\
\text { right" }\end{array}$} & \\
\hline & A15 & & \\
\hline & A16 & \multirow{2}{*}{\begin{tabular}{|c|} 
Links between goal \\
nodes and action \\
nodes \\
11: Going straight, \\
10: Turn left, 01: \\
Turn right
\end{tabular}} & \multirow[b]{2}{*}{$\begin{array}{l}\text { External links } \\
\text { (from goals) }\end{array}$} \\
\hline & A17 & & \\
\hline
\end{tabular}




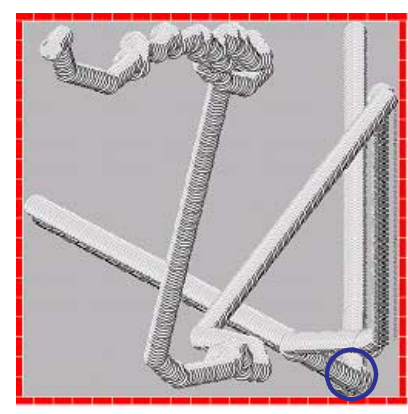

(a) Before learning

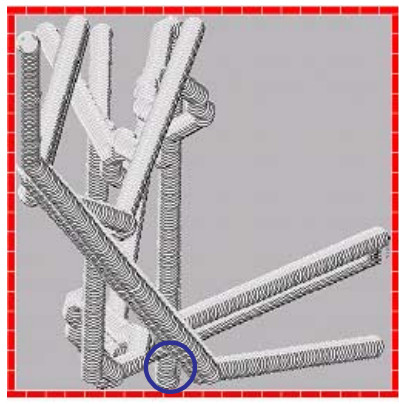

(c) Before learning

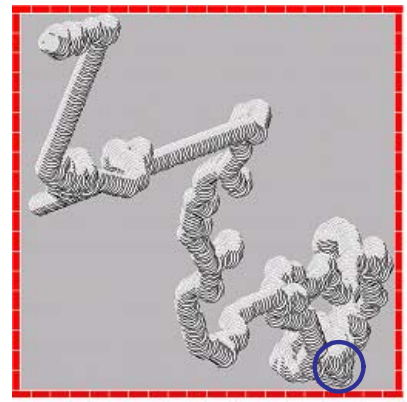

(b) After learning

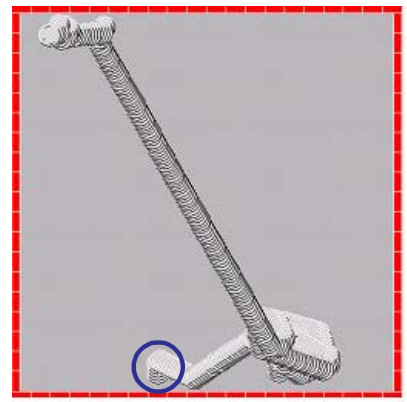

(d) After learning

Fig. 8. Action results according to different initial positions of the robots

action of going straight were selected. Fig. 8 shows the results of learning when the initial position of robot is same. While in Fig. 8(a) the robot reached the goal in 5533 steps, it reached the goal in 1457 steps after learning (see Fig. 8(b)). Also as shown in Fig. 8(c) and Fig. 8(d), while the robot reached goal in 5435 steps before learning, it reached in 448 steps after learning.

Fig. 9 shows an example of the networks with higher fitness after robot's learning. As comparing with the initial network (see Fig. 7), learning procedures make the links between nodes adapt to the changes of task. Firstly the robot moved only by going straight, but after learning it moved by selecting the actions of reaching the goal faster as shown as Fig. 8 and Fig. 9.

The learning mode performance measures how well the LCS is learning to perform the correct behavior in an environment. In order to evaluate its performance, we use the simple measurement of learning performance, the ratio of the number of correct responses during epoch to the epoch length, as follows [14]:

\section{$\frac{\text { Number of correct responses during epoch }}{\text { Epoch length }}$}

Fig. 10 shows the average number of correct responses at every epoch. It proves that the robot can select appropriate behaviors via learning. Table 3 shows the average steps of robot's reaching the goal. It means the robot reached the goal much faster after learning. 


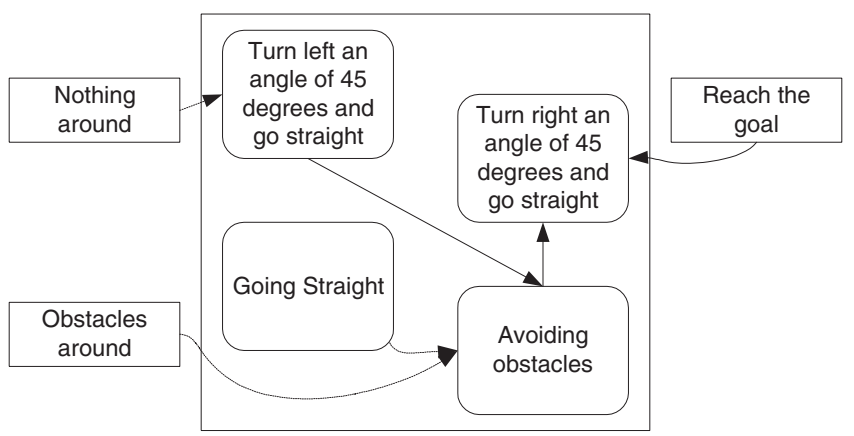

(a)

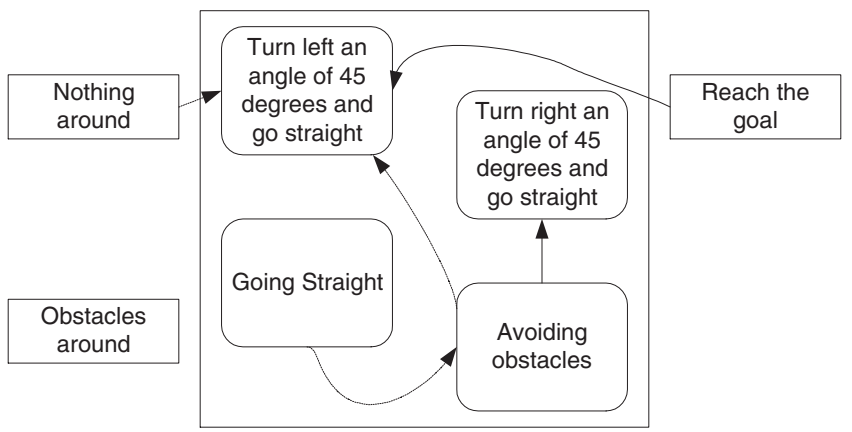

(b)

Fig. 9. Changes in action selection network after learning: Dotted lines mean inhibitory links and solid lines mean excitatory links in the internal links

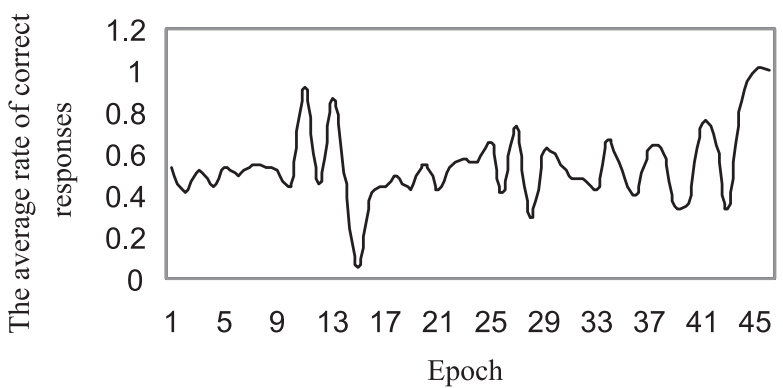

Fig. 10. The average rate of correct responses with respect to epochs 
Table 3. The average steps of robot's reaching the goal before and after learning

\begin{tabular}{|c|c|c|}
\hline & Before learning & After learning \\
\hline Average steps & 7936.7 & 4421.8 \\
\hline
\end{tabular}

\section{Conclusions}

In this paper, Maes' action selection network is the object for learning. It has a weakness of redesigning the network structure when the task changes. That is, it is impossible to modify the network defined in the beginning. The network needs tobe reorganize its structure according to the given tasks. In order to do this, we applied the idea of learning to the network. As a result, we verified that the robot reorganized the network structure by making the rules appropriate to the changing states, and the robot moved successfully. Besides the robot can select more correct behaviors through learning. However, we used very simple model that consisted of only four actions in order to show the usefulness of learning the action selection network. Further works are going on studying more complicated network with more various nodes that adapts to changes.

\section{Acknowledgements}

This work was supported by Korea Research Foundation Grant (2002-005H20002).

\section{References}

[1] P. Maes, and R. Brooks, "Learning to Coordinate Behaviors," Proceedings AAAI90: the American Conference on Artificial Intelligence, Boston, MA, pp.796-802, 1990. 578

[2] K.-J. Kim, S.-B. Cho, and S.-R.Oh, "Behavior Selection Architecture for Tangible Agent," IEEE Internationl Symposium on Computational Intelligence in Robotics and Automation, 2003. 578

[3] N. Tinbergen, The Study of Instincts, Oxford, UK: Oxford Univ. Press, 1996. 578

[4] R. A, Brooks, "A Robust Layered Control System for a Mobile Robot," IEEE Journal of Robotics and Automation, pp. 14-23, 1986. 578

[5] T. Tyrrell, "An Evaluation of Maes' Bottom-up Mechanism for Bahavior Selection," Adaptive Behavior, vol. 2, pp. 307-348, 1994. 578

[6] M. J. Mataric, "Behavior-Based Control: Examples from Navigation, Learning, and Group Behavior," Journal of Experimental and Theoretical Artificial Intelligence, Special Issue on Software Architectures for Physical Agents, pp. 323-336, 1997. 578

[7] R. Arkin, Behavior-Based Robotics, MIT Press, Boston, MA, 1998. 578

[8] S. Mahadevan, and J. Connell, "Scaling Reinforcement Learning to Robotics by Exploiting the Subsumption Architecture," Eighth International Workshop on Machine Learning, Morgan Kaufmann, pp. 328-337, 1991. 578 
[9] M. Dorigo, and M. Colombetti, Robot Shaping: An Experiment in Behavior Engineering, MIT Press, MA, 1997. 578

[10] P. Maes, "How To Do the Right Thing," Connection Science Journal, vol. 1, no. 3, pp. 291-323, 1989. 579

[11] D. E. Goldberg, Genetic Algorithms in Search, Optimization, and Machine Learning, Addison-Wesley, MA, 1989.

[12] L. Booker, D. E. Goldberg, and J.H. Holland, "Classifier Systems and Genetic Algorithms," Artificial Intelligence, vol. 40, pp. 235-282. 1989. 580, 582

[13] O. Michel, "Khepera Simulator Package version 2.0: Freeware Mobile Robot Simulator Written by Oliver Michel," http://diwww.epfl.ch, 1996. 583

[14] R. A. Richards, "Zeroth-Order Shape Optimization Utilizing a Learning Classifier System," Ph. D. Dissertation, Mechanical Engineering Department, Stanford University, 1995. 586 